\title{
PERFIL DO DESENVOLVIMENTO MOTOR DE ALUNOS DE OITO ANOS DE ESCOLA PUBLICAS ESTADUAIS DE SÃO BENTO DO SUL - SC
}

\author{
Angelita de Souza*, William Cordeiro de Souza*, Fernando Carvalheiro Reiser**, Marcio Cascante \\ Rusenhack ${ }^{* * *}$, José Moncada-Jiménez ${ }^{* * *}$, Luis Paulo Gomes Mascarenhas ${ }^{* * * *}$ \\ Autor correspondente: William Cordeiro de Souza. E-mail: williammixx@hotmail.com \\ * Universidade do Contestado - UnC. Especialista em Fisiologia do Exercício com Enfase em treinamento Esportivo \\ * Universidade do Vale do Itajaí - Univali. \\ *** Universidade da Costa Rica - UCR \\ **** Professor da Universidade Estadual do Centro-Oeste - Unicentro.
}

\begin{abstract}
Resumo
Introdução: O desenvolvimento motor classifica-se como um processo de mudança sequencial no nível de funcionamento do indivíduo que engloba uma série de transformações em seu comportamento motor. Objetivo: Traçar o perfil de desenvolvimento motor de alunos de oito anos das escolas públicas estaduais da cidade de São Bento do Sul - SC. Métodos: A amostra constituiu-se de 50 alunos de ambos os gêneros; para a pesquisa foi utilizado como instrumento de coleta de dados a Escala de Desenvolvimento Motor (EMD), desenvolvida por Rosa Neto (2002), a qual avalia o desenvolvimento motor do indivíduo, testando os parâmetros de motricidade fina, motricidade global, equilíbrio, organização espacial e temporal e esquema corporal. A análise descritiva dos dados foi feita através da média, desvio padrão, valor mínimo, valor máximo. Resultados e Conclusão: Considerando os resultados obtidos no presente estudo, pode-se concluir que os alunos apresentaram um perfil de desenvolvimento motor dentro da normalidade, ou seja, compatíveis com a idade cronológica em que se encontravam. Palavras Chave: Desenvolvimento motor; Avaliação motora; Idade motora.
\end{abstract}




\title{
PROFILE OF MOTOR DEVELOPMENT IN PUBLIC STATE SCHOOL'S EIGHTH GRADE STUDENTS IN SÃO BENTO DO SUL - SC
}

\begin{abstract}
Introduction: Motor development is classified as a sequential process of change in the individual's level of functioning which includes a series of changes in their motor behavior. Objective: This study aimed intended to draw the eight-year students of motor development profile of public schools in São Bento do Sul - SC. Methods: The sample consisted of 50 students of both genders; for the research was used as a tool for data collection Motor Development Scale (EMD) developed by Rosa Neto (2002), which evaluates the individual's motor development, testing the parameters of fine motor skills, overall motor skills, balance, organization spatial and temporal and body image. A descriptive analysis was performed by mean, standard deviation, minimum value, maximum value. Results and Conclusion: Considering the results obtained in this study, it can be concluded that the students had a motor development profile within the normal range, ie compatible with chronological age in which they were.
\end{abstract}

Keywords: Motor Development; Motor assessment; Motor age.

\section{INTRODUÇÃO}

O desenvolvimento motor é conceituado como um processo de mudança que engloba um conjunto de transformações no comportamento motor, ou seja, a criança adquire uma capacidade de controlar seus movimentos ao longo do tempo. ${ }^{(1,2)}$

Esse desenvolvimento refere-se às mudanças que ocorrem na capacidade motora, onde a criança desencadeia interação com o seu ambiente e com a tarefa que ela esteja executando. ${ }^{(3,4)}$ Cabe ressaltar que o processo evolutivo biológico tem grande influencia nos fatores ambientais e sociais, e podem influenciar o desenvolvimento motor. ${ }^{(5)}$

Para Haywood; Getchell(6) esse processo é considerado como sequencial, contínuo e relacionado à idade cronológica, pelo qual o ser humano adquire uma vasta gama de habilidades motoras, as quais progridem de movimentos simples e desorganizados para a execução de movimentos organizados e complexos.
O desenvolvimento é um processo extenso, que inicia com os movimentos reflexos progredindo para movimentos rudimentares e fundamentais e por fim para a fase dos movimentos especializados, onde se dividem em três estágios: o transitório, o de aplicação e o de utilização permanente. ${ }^{(3)}$

Vale ressaltar que no início do processo de escolarização ocorre um amplo incremento nas habilidades motoras, pois a escola, especificadamente as aulas de educação física (EDF) possibilita a criança um amplo domínio do seu corpo em diferentes atividades, como: saltar, correr, rastejar, chutar uma bola, arremessar um arco, equilibrar-se num pé só, escrever, entre outras. ${ }^{(7,8)}$

A EDF apresenta um papel importante, pois oferece experiências, resultando assim numa grande auxiliar e promotora do desenvolvimento humano, em especial ao desenvolvimento motor e garanti- 
ra aprendizagem de habilidades específicas nos jogos, esportes, ginásticas e dança. ${ }^{(1)}$

Sendo assim, o presente estudo buscou como objetivo traçar o perfil de desenvolvimento motor de alunos de oito anos das escolas públicas estaduais da cidade de São Bento do Sul - SC.

\section{MATERIAIS E MÉTODOS}

O presente estudo caracterizou-se como investigativo e diagnóstico, e sua amostra foi constituída por 50 alunos de oito anos de idade cronológica de ambos os gêneros, sendo 24 meninas e 26 meninos, correspondente ao $3^{\circ}$ ano do Ensino Fundamental. Os avaliados pertenciam a três escolas da zona urbana da rede pública estadual da cidade de São Bento do Sul - SC.

Os pais e os responsáveis pelos os alunos receberam um termo de consentimento para ser preenchido, no qual continha uma breve explicação dos objetivos e dos procedimentos metodológicos do estudo. Foram excluídas do estudo crianças que apresentaram doenças crônicas ou específicas do crescimento, que não compareceram na escola nos dias marcados para coleta de dados e as crianças cujos pais não autorizaram a participação. Sendo também respeitada a vontade das crianças que se recusaram a participar mesmo com a autorização dos pais. Esta pesquisa seguiu os princípios éticos de respeito à autonomia das pessoas, apontada pela Resolução n 196, de 10 de outubro de 1996 do Conselho Nacional de Saúde. É valido ressaltar que este trabalho foi aprovado pelo Comitê de Ética da Universidade do Contestado - UnC (número do Parecer: CAAE: 03914912.2.0000.0117).
Os testes foram aplicados com base na Escala de Desenvolvimento Motor (EDM) proposta por Rosa Neto, ${ }^{(9)}$ a mesma apresenta confiabilidade em avaliar o desenvolvimento motor de escolares, ${ }^{(7)}$ a escala é definida pelos termos idade cronológica (dada a partir da data de nascimento convertida em meses); idade motora geral (soma dos resultados obtidos em seis baterias de testes, abrangendo as seguintes áreas da motricidade humana): idade motora um (motricidade fina); idade motora dois (motricidade global); idade motora três (equilíbrio); idade motora quatro (esquema corporal); idade motora cinco (organização espacial) e idade motora seis (organização temporal).

Consistem em 10 tarefas motoras cada, distribuídas entre dois e onze anos, organizadas progressivamente em grau de complexidade. $O$ teste inicia a partir da idade cronológica da criança (IC); se a mesma conseguiu realizar o teste correspondente à sua idade atribui-se um valor correspondente a idade motora (IM) expressa em meses, em caso de êxito, a avaliação em cada área continua até a idade motora que o aluno conseguiu chegar. Possui também testes de lateralidade com metodologia diferenciada.

Ao final da aplicação, dependendo do desempenho individual em cada bateria, é calculada a idade motora geral (IMG) que é a soma dos resultados obtidos nos testes e esta soma é então dividida pelo numero de testes (total de seis), obtendo o quociente motor geral (QMG) da criança. O valor do quociente motor é obtido pela divisão entre a idade cronológica multiplicada por 100. Esses valores são quantificados e categorizados conforme a quadro 1.

Quadro 1 - Classificação do quociente motor em meses.

\begin{tabular}{|c|c|}
\hline 130 ou mais & Muito superior \\
\hline $120-129$ & Superior \\
\hline $110-119$ & Normal Alto \\
\hline $90-109$ & Normal médio \\
\hline $80-89$ & Normal baixo \\
\hline $70-79$ & Inferior \\
\hline 69 ou menos & Muito inferior \\
\hline
\end{tabular}

Fonte: Rosa Neto ${ }^{(9)}$ 
Para o tratamento estatístico foi utilizado o programa Excel. Para a análise dos dados foi utilizada a estatística descritiva através da média, desvio padrão, valor mínimo e máximo. Para a lateralidade foi utilizado à inferência percentual.

\section{RESULTADOS}

De acordo com o objetivo da pesquisa, de traçar o perfil do desenvolvimento motor de alunos de oito anos, os resultados apresentam a análise descritiva das variáveis, idade cronológica (IC), idade moto- ra (IMG), quociente motor referente à motricidade fina ( $\left.\mathrm{QM}_{1}\right)$, quociente motor referente à motricidade global $(\mathrm{QM} 2)$, quociente motor referente a equilíbrio ( $\mathrm{QM} 3)$, quociente motor referente a esquema corporal (QM4), quociente motor referente à organização espacial (QM5), quociente motor referente à organização temporal (QM6) e quociente motor geral (QMG). Na tabela 1 estão expressos os resultados das variáveis estudadas dos alunos. Em relação às características motoras, verifica-se que a IC média da amostra foi de 101,39 meses, com IMG de 106 meses.

Tabela 1 - Distribuição do comportamento das variáveis da avaliação motora.

\begin{tabular}{lcccc}
\hline \multicolumn{1}{c}{ VARIÁVEIS } & MÉDIA & $\begin{array}{c}\text { DESVIO } \\
\text { PADRÃO }\end{array}$ & $\begin{array}{c}\text { VALOR } \\
\text { MínIMO }\end{array}$ & $\begin{array}{c}\text { VALOR } \\
\text { MÁXIMO }\end{array}$ \\
\hline Idade Cronológica - IC & 101,39 & $+3,57$ & 94 & 108 \\
Idade motora -IMG & 106,00 & $+7,34$ & 88 & 124 \\
Motricidade fina - QM1 & 103,33 & $+11,70$ & 82 & 130 \\
Motricidade global - QM2 & 106,17 & $+4,49$ & 92 & 116 \\
Equilíbrio - QM3 & 101,11 & $+12,65$ & 73 & 131 \\
Esquema Corporal - QM4 & 106,00 & $+15,67$ & 70 & 137 \\
Organização espacial- QM5 & 105,07 & $+14,72$ & 80 & 139 \\
Organização temporal - QM6 & 108,41 & $+12,47$ & 82 & 139 \\
Quociente motor geral - QMG & 104,67 & $+7,91$ & 89 & 122 \\
\hline
\end{tabular}

Nota: As idades são apresentadas em meses.

Ao analisar a relação entre a IC e a IMG dos alunos, observou-se que a IMC sobre a IC apresentou índice superior em cinco meses, diferença esta aceitável, com base no instrumento de Rosa Neto, ${ }^{(9)}$ utilizado, para o que se espera com relação a sua idade cronológica. Por analise geral do grupo, todas as áreas motoras avaliadas obtiveram classificação normal médio (QM entre 90 a 109).
A tabela 2 apresenta as classificações da IMG dos alunos avaliados, onde $76 \%(n=38)$ apresentam a IMG compatível com a IC, e $6 \%(\mathrm{n}=3)$ mostraram estar dentro da classificação normal baixa, e $14 \%(n=7)$ normal alta, apenas $4 \%(n=2)$ dos avaliados obteve como resultado a IM superior à IC e nenhuma apresentou idade motora inferior.

Tabela 2 - Frequência da classificação geral dos resultados do índice motor geral.

\begin{tabular}{lcc}
\hline \multicolumn{1}{c}{ TABela De ClaSSIFICAÇÃO } & $\mathrm{N}=$ & $\%$ \\
\hline Normal Baixo & 3 & $6 \%$ \\
Normal Médio & 38 & $76 \%$ \\
Normal Alto & 7 & $14 \%$ \\
Superior & 2 & $4 \%$ \\
Total & 50 & $100 \%$ \\
\hline
\end{tabular}


A tabela 3 apresenta os resultados de lateralidade dos alunos avaliados, evidenciou-se que $96 \%$ possuem lateralidade definida, destes $64 \%(n=32)$ são destros completos, ou seja, apresentam lateral direita de mãos, olhos e pés, $4 \%(n=2)$ sinistro completo e $28 \%(n=14)$ apresentam lateralidade cruzada (arremessam com a mão direita e chutam com o pé esquerdo, por exemplo). E apenas $4 \%(n=2)$ dos alunos apresentaram lateralidade indefinida.

Tabela 3 - Frequência percentual da avaliação de lateralidade.

\begin{tabular}{lcc}
\hline \multicolumn{1}{c}{ LATERALIDADE } & $\mathrm{N}=$ & $\%$ \\
\hline Cruzada & 14 & 28 \\
Sinistra & 2 & 4 \\
Destro Completo & 32 & 64 \\
Indefinida & 2 & 4 \\
Total & 50 & 100 \\
\hline
\end{tabular}

\section{DISCUSSÃO}

O presente trabalho buscou como objetivo traçar o perfil de desenvolvimento motor de alunos de oito anos e verificou que os alunos apresentaram um perfil de desenvolvimento motor dentro da normalidade, ou seja, compatíveis com a idade cronológica em que se encontravam. A média dos valores encontrados neste estudo para $\mathrm{QMI}, \mathrm{QM}_{4}, \mathrm{QM}_{5}$, e QM6 foi de normal médio, conforme a classificação da EDM.

Esses dados condizem com os encontrados por Rosa Neto(9) em um estudo realizado com 75 alunos de $1^{\mathrm{a}}$ à $4^{\mathrm{a}}$ séries do ensino fundamental. Já os valores médios obtidos de $\mathrm{QM} 2=106,17$ e QM3= 101,11 mostram-se dentro da classificação EDM de normal média. A média da IMG neste estudo é de 106 meses, quando comparando com o estudo acima supracitado, o presente trabalho apresentou valores foi acima dos valores encontrados que foram 95,03, mas vale ressaltar que ambos os estudos estão dentro da classificação normal médio.

Apenas $4 \%$ da amostra, apresentaram superioridade na classificação, lembrando que o indivíduo se desenvolve em ritmos diferentes, dependem de suas próprias características e das experiências vivenciadas e possibilita que diferentes padrões sejam detectados. ${ }^{(10)}$
Outro ponto a ser analisado, Rosa Neto e ou$\operatorname{tros}^{(7)}$ descreve que a lateralidade tendo como parte integrante da estruturação espacial e a desorientação espacial pode estar relacionada a presença da lateralidade cruzada, a amostra apresentada na pesquisa do autor $1 / 3$ da lateralidade cruzada apoiando a baixa classificação que obteve na orientação espacial. Resultado este, diferente da presente pesquisa que aponta $28 \%$ da lateralidade cruzada e a média da organização espacial mostra um índice normal médio de 105,7. Sendo que, $64 \%(n=32)$ da amostra apresentam sua lateralidade definida, predominando a preferência da lateral direita.

Amaro e outros ${ }^{(10)}$ com objetivo de avaliar o desenvolvimento motor de crianças com dificuldades na aprendizagem escolar verificaram que $76,3 \%$ dos escolares apresentaram índice de desenvolvimento "inferior" e "muito inferior", com média do QMG de 69,50 (inferior). Dados esses que se contrapõe aos achados do presente estudo. Os autores ainda relatam que os dados encontrados no estudo apresentam relação entre déficits motores e dificuldades na aprendizagem.

Rosa Neto e outros ${ }^{(10)}$ com o intuito de avaliar o desenvolvimento motor de crianças com indicadores de dificuldades na aprendizagem escolar verificaram que de acordo com a classificação da EDM, $74,3 \%$ os escolares apresentaram índice de "Nor- 
mal Médio" à "Normal Baixo" com média do QMG de 89,48 (normal baixo). Do total de escolares avaliados, $87,1 \%(n=27)$ apresentou média de 16 meses de atraso motor. Dados esses que se contrapõe aos achados do presente estudo.

Porta e outros ${ }^{(4)}$ avaliaram o desenvolvimento motor de crianças eutróficas e obesas e verificaram que a classificação de ambos os grupos esteve dentro da normalidade (normal médio), o grupo de obesos apresentou resultados inferiores ao grupo de eutróficos em todas as áreas, com diferença significante no desenvolvimento motor geral, na motricidade global, no equilíbrio e no esquema corporal. Os resultados indicam que algumas crianças obesas apresentaram dificuldades motoras.

Dados semelhantes ao presente estudo foram encontrados por Soares e outros ${ }^{(11)}$ que verificaram o perfil motor dos escolares do Ensino Fundamental I do Município de Chapecó, SC, a amostra foi composta por 91 escolares de ambos os gêneros na faixa etária de 6 a 10. No geral, os resultados apontam que a média da IC dos escolares participantes ficou em 94,01 meses, a média da IMG foi de 94,79 meses e o QMG foi de 101,38, o que classificou os escolares em um perfil motor normal médio. Quando analisados por habilidades, os resultados apontam que somente no esquema corporal, organização espacial e estrutura temporal os escolares apresentaram uma idade motora abaixo da média de IC. No que diz respeito à lateralidade, a maioria dos escolares apresentou uma prevalência destra.

Como mencionado anteriormente, o presente estudo limitou-se em não controlar o estado nutricional dos alunos avaliados para verificar a existência de relação com os resultados obtidos na EDM. Outro fator limitante foi o numero de sujeitos avaliados, sendo uma amostra pequena para três escolas. Dessa forma, sugere-se que para futuros estudos nesta faixa etária, controle-se o estado nutricional com o intuito de entender as possíveis dificuldades motoras encontradas em crianças com excesso de peso. Porta et al.(4) destacam crianças obesas apresentaram dificuldades motora.

\section{CONCLUSÃO}

A partir dos resultados obtidos foi possível constatar que o desenvolvimento da motricidade fina, motricidade global, equilíbrio, esquema corporal e organização espacial dos alunos avaliados estão dentro do recomendado. De modo geral, o presente estudo mostra que o desenvolvimento motor dos avaliados encontra-se dentro dos parâmetros da normalidade, ou seja, compatíveis com a idade cronológica em que se encontravam.

\section{REFERÊNCIAS}

1. Rosa Neto FR, Almeida GMF, Caon G, Ribeiro J, Caram JA, Piucco EC. Desenvolvimento Motor de Crianças com Indicadores de Dificuldades na Aprendizagem Escolar. Rev. Bras. Ci e Mov. 2007;16(1):45-51.

2. Silva MNS, Dounis AB. Perfil do desenvolvimento motor de crianças entre 9 e 11 anos com baixo rendimento escolar da rede municipal de Maceió, AL. Cad. Ter. Ocup. 2014;22(1) 63-70.

3. Gallahue DL, Ozmun JC. Compreendendo o desenvolvimento motor: bebês, crianças, adolescentes e adultos. $3^{a}$ ed. São Paulo - SP: Phorte; 2005.

4. Poeta LS, Duarte MFS, Giuliano ICB, Silva JC, Santos APM, Rosa Neto F. Desenvolvimento motor de crianças obesas. Rev. Bras. Ci. e Mov. 2010;18(4):18-25.

5. Santos AM, Rosa Neto F, Pimenta RA. Avaliação das habilidades motoras de crianças participantes de projetos sociais/esportivos. Motricidade. 2013;9(2):51-61.

6. Haywood KM, Getchell N. Desenvolvimento motor ao longo da vida. $3^{a} \mathrm{Ed}$ : Porto Alegre RS: Artmed; 2004.

7. Rosa Neto F, Santos ANM, Xavier RFC, Amaro KN. A importância da avaliação motora em escolares: análise da confiabilidade da escala de desenvolvimento motor. Rev. Bras. Cineantropom. Desempenho Hum. 2010;12(6):422-7. 
8. Cordeiro S, Souza WC, Robles AR, Calliari ICR, Grzeczak MT, Mascarenhas LPG. et al. Estudo comparativo do crescimento físico e desenvolvimento motor entre crianças de 10 anos de idade, residentes no perímetro urbano e área rural do município de São João do Triunfo - PR. Rev. Pulsar. 2014;6(2):1-11.

9. Rosa Neto F. Manual de avaliação motora. $1^{\text {a }}$ Ed: Porto Alegre - RS: Artmed; 2002.
10. Amaro KN, Jatobá L, Santos APM, Rosa Neto F. Desenvolvimento motor em escolares com dificuldades na aprendizagem. Movimento \& Percepção. 2010;11(16):39-47.

11. Soares RAOS, Vieira MP, Oliveira SR, Werhauser S. Perfil motor de escolares da rede pública do município de Chapecó, SC. Unoesc \& Ciência. 2015;(Ed. Esp):79-86. 